\title{
EchoGéo
}

29 | 2014

The Political Ecology of Conservation and

Development Territories

\section{Encadrement des plantations d'eucalyptus à Addis Abeba : vers une confrontation des discours environnementaux et des pratiques populaires}

Delphine Ayerbe

\section{(Q) OpenEdition}

Journals

Electronic version

URL: https://journals.openedition.org/echogeo/13936

DOI: 10.4000/echogeo.13936

ISSN: 1963-1197

Publisher

Pôle de recherche pour l'organisation et la diffusion de l'information géographique (CNRS UMR 8586)

Electronic reference

Delphine Ayerbe, "Encadrement des plantations d'eucalyptus à Addis Abeba : vers une confrontation des discours environnementaux et des pratiques populaires", EchoGéo [Online], 29 | 2014, Online since 01 December 2014, connection on 31 July 2021. URL: http://journals.openedition.org/echogeo/13936 ; DOI: https://doi.org/10.4000/echogeo.13936

This text was automatically generated on 31 July 2021.

EchoGéo est mis à disposition selon les termes de la licence Creative Commons Attribution - Pas d'Utilisation Commerciale - Pas de Modification 4.0 International (CC BY-NC-ND) 


\title{
Encadrement des plantations d'eucalyptus à Addis Abeba : vers une confrontation des discours environnementaux et des pratiques populaires
}

\author{
Delphine Ayerbe
}

\section{Introduction}

1 Depuis son introduction en Éthiopie il y a un peu plus d'un siècle, l'eucalyptus a été diffusé massivement sur le territoire national. Addis Abeba, la capitale, a notamment adopté cette espèce exotique et est aujourd'hui plongée dans une forêt urbaine et périurbaine. Ces plantations alimentent un marché local toujours demandeur de bois de chauffe et de bois d'œuvre. Le gouvernement fédéral et la Municipalité se partagent la très grande majorité des exploitations. Mais le succès économique de ces plantations, et la demande sociétale qui le supporte, est entravé par un rejet écologique de l'espèce. Accusé d'assécher les sols et de favoriser l'érosion des collines de la capitale, l'eucalyptus pourtant si présent dans le paysage additien, devient l'élément central d'un vif débat qui anime les décideurs urbains.

2 La ville est actuellement le théâtre de profondes mutations. Un élan de modernisation emporte les aménageurs qui multiplient la mise en place de chantiers d'envergure (infrastructures, logements, etc...). Un travail est fait pour valoriser l'image de cette capitale et affirmer son positionnement sur la scène internationale. Addis Abeba doit devenir la vitrine d'un pays qui se modernise et qui rompt avec les sombres images des années 1980 (Gascon, 2008). Dans ce contexte particulier, la place des exploitations d'eucalyptus dans le paysage urbain est critiquée. Ce rejet nous semble dépasser le strict cadre des problématiques environnementales, que posent en premier lieu les 
représentants institutionnels. Ainsi nous tenterons de comprendre les objectifs plus larges qui motivent cette approche nouvelle des espaces boisés. Et surtout de saisir les enjeux et les intérêts portés par les discours environnementaux en vogue.

3 L'étude s'inscrit dans les recherches sur l'environnement urbain dans les pays du Sud et notamment dans la continuité des travaux menés sur ce thème par l'IRD. Dans le contexte de mutations que connaît Addis Abeba, un regard particulier est porté sur l'environnement. Sa valorisation devient un élément de marketing urbain, et permet de travailler l'image d'une ville «durable» (Couret, 2011; Metzger, 2010). Les préceptes nouveaux qui sont posés par les décideurs urbains signalent une appropriation d'arguments environnementaux édités par les pays du Nord ${ }^{1}$. Cette influence, souvent coercitive, a été analysée avec précision dans les contextes ruraux par Georges Rossi (2001). La prise en compte croissante des questions environnementales en milieu urbain s'inscrit alors dans une référence aux changements globaux (Metzger, 1994).

4 Notre objet d'étude s'avère une entrée intéressante pour saisir les nouvelles pratiques environnementales à l'œuvre dans cette métropole africaine. Les espaces plantés d'eucalyptus cristallisent des tensions nouvelles qui nous renseignent sur les orientations et les préoccupations des autorités publiques. Les réflexions engagées autour de leur place dans le paysage urbain mêlent des arguments écologiques, économiques, mais aussi - plus étonnamment - identitaires et patrimoniaux. Surtout, l'étude des modalités de gestion et de contrôle des plantations d'eucalyptus à Addis Abeba permet de saisir un rapport de force nouveau entre un gouvernement puissant et internationalisé, et des pratiques populaires décriées.

5 Les spécificités du contexte éthiopien offrent aux autorités une large palette d'outils de régulation (Belachew, 2010). Le système foncier favorise l'État, qui est l'unique propriétaire du sol, et conforte ainsi une assise politique par son ancrage territorial. Nous tenterons donc de déceler les rouages de cette nouvelle donne environnementale en questionnant les exigences posées par la gestion des plantations d'eucalyptus. Le retour sur les termes du rejet de l'espèce nous montrera l'influence, entre autre, des discours globaux dans les choix de préservation de l'environnement. Nous illustrerons ensuite notre propos par deux exemples où s'exercent des modalités nouvelles de régulation d'accès à la ressource par les autorités en charge de ces espaces. Enfin nous interrogerons les motivations des acteurs impliqués dans l'élaboration de tentatives de normalisation des espaces plantés.

\section{L'eucalyptus : « une commodité problématique »²}

\section{Les reboisements en eucalyptus à Addis Abeba}

6 La capitale éthiopienne est plongée au cœur d'une forêt urbaine qui résulte de plus d'un siècle de reboisements en eucalyptus. Lorsqu'Addis Abeba a été fondée à la fin du XIX siècle sur les hauts plateaux du Choa par l'empereur Ménélik, les ressources boisées nécessaires au développement de la ville ont rapidement manqué (Gascon, 1999). L'introduction de cette espèce exotique, sur recommandation d'un conseiller étranger présent à la cour, a permis de résoudre les pénuries en bois de chauffe et bois d'œuvre et a facilité la fixation de la nouvelle capitale éthiopienne. Dès lors, l'espèce a connu un vif succès, auprès des gouvernements successifs comme des populations locales. 
7 De croissance rapide, l'eucalyptus a connu une diffusion massive dans l'espace additien - mais aussi à l'échelle du pays. Des distributions de semences orchestrées par le gouvernement dès la fin du XIX siècle aux vastes programmes de reboisement des années 1980, les incitations au développement des plantations ont été nombreuses. Aujourd'hui environ 8000 ha de plantations recouvrent le territoire urbain. Les plantations massives d'eucalyptus, comme les exploitations individuelles plus modestes, sont venues reverdir, en un peu plus d'un siècle, des espaces largement dénués de végétation. Les gouvernements successifs ont été à l'initiative de ces reboisements. Si l'empereur Ménélik a favorisé la distribution de graines aux nouveaux habitants, le régime d'Hailé Sélassié a octroyé d'immenses réserves foncières à la noblesse impériale sur lesquelles les eucalyptus ont eu une place de choix (Berlan, 1963). La ressource boisée a permis une mise en valeur rapidement rentable de ces terres pour un petit nombre de grands propriétaires terriens. La prise du pouvoir en 1974 par la junte militaire du Derg a renversé les rapports d'accès au foncier en imposant dès 1975 une nationalisation des terres. Le pouvoir central, maître en sa demeure, a organisé dans la seconde partie des années 1980, de grandes campagnes de reboisement, avec le soutien d'organisations internationales. Ces campagnes ont fait suite aux famines tragiques et fort médiatisées des années 1984-85 qui ont touché l'Éthiopie. Le regard posé par la communauté internationale sur le pays a soutenu l'élaboration de projets de développement. L'accent a été mis sur l'amélioration des disponibilités en ressources naturelles. Ces grandes famines marquent un tournant dans les politiques environnementales de l'État éthiopien. Des programmes de réhabilitation et de conservation des sols sont instaurés en vue d'améliorer la sécurité alimentaire (Keeley, 2000 ; Amare, 2003). Les campagnes de reboisement - même en eucalyptus - s'inscrivent dans cette dynamique. Elles ont été marquées par une intensité particulière tant spatiale que temporelle. Elles ne se sont pas arrêtées aux portes de la capitale, bien au contraire, et ont été menées dans diverses zones rurales et villes secondaires, où les eucalyptus - suivant le modèle de mise en valeur éprouvé par Addis Abeba - étaient déjà présents. À la chute du Derg en 1991, leur gestion est passée aux mains du nouveau gouvernement. À Addis Abeba les plantations sont gérées pour une partie par la Municipalité, et pour l'autre par l'entreprise étatique Finfinnee Forest Development and Marketing Enterprise. Ce partage de gestion se fait au niveau des frontières de la région-capitale. La domination des espaces plantés au nord de la capitale (illustration 1), est directement héritée de programmes environnementaux du Derg. Ces collines, à immédiate proximité du centre urbain, représentaient des lieux privilégiés pour déployer ces programmes de reboisement ${ }^{3}$. 


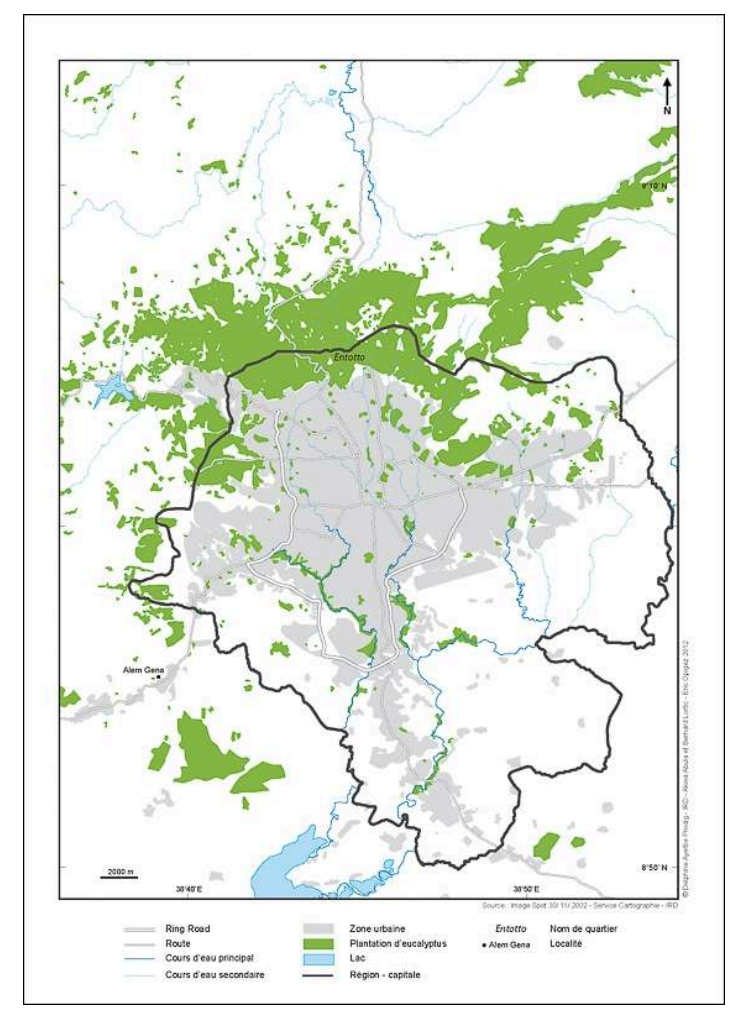

Source : Image Spot : 30/11/2002 ; service cartographique : IRD - auteurs : D. Ayerbe, A. Abula, B. Lortic, E. Opigez, 2012.

8 Ainsi, au cours du dernier siècle, l'espèce exotique s'est enracinée dans le paysage éthiopien. Elle s'est hissée au rang de ressource boisée indispensable. Ses usages divers comme bois de chauffe, de cuisson, de construction (habitation ou montage d'échafaudage) ont valu à l'eucalyptus d'être approprié par les populations. Cet arbre est devenu l'élément constitutif d'une filière agricole solide qui réunit une large palette d'acteurs, du grand exploitant au petit revendeur. De plus, l'espèce a été diffusée dans les espaces domestiques, individuels. Il est rare de trouver un jardin privé qui n'abrite pas trois ou quatre eucalyptus, dont la coupe occasionnelle peut servir à fournir le bois nécessaire à l'organisation de grands évènements familiaux (repas de fête, etc...). Le travail de terrain réalisé en 2009-10 à Addis Abeba atteste de cet attachement populaire à l'arbre urbain. Un premier temps d'observation nous a permis de constater l'omniprésence de l'usage du bois dans les foyers additiens. Nous avons réalisé une série d'entretiens pour tenter de cerner cette utilisation de l'arbre chez les habitants. Nous avons sélectionné trois quartiers différents : un quartier d'habitat très populaire du centre-ville (maisons de bois et toits de tôles); un quartier de classe moyenne (immeubles condominiums) et un quartier de pavillons en périphérie. Dans chaque site nous avons réalisé entre 5 et 10 entretiens qui ont permis de confirmer cet attachement à l'usage du bois de chauffe et de cuisson que nous avions supposé. Mais contrairement à ce que nous envisagions, le revenu des foyers ne s'est pas avéré le seul facteur explicatif. Dans les quartiers pavillonnaires où la population est relativement aisée l'usage de l'eucalyptus n'est pas marginal. Si le bois permet de pallier les fréquentes coupures d'électricité, il s'impose aussi pour son usage dans la cuisine traditionnelle. 
Ses usages constants soutiennent la production de ressources boisées. Ainsi, comme le montre l'illustration 2, la ville reste plongée au cœur d'une forêt, recréée pour la servir.

Illustration 2 - Vue d'Addis Abeba

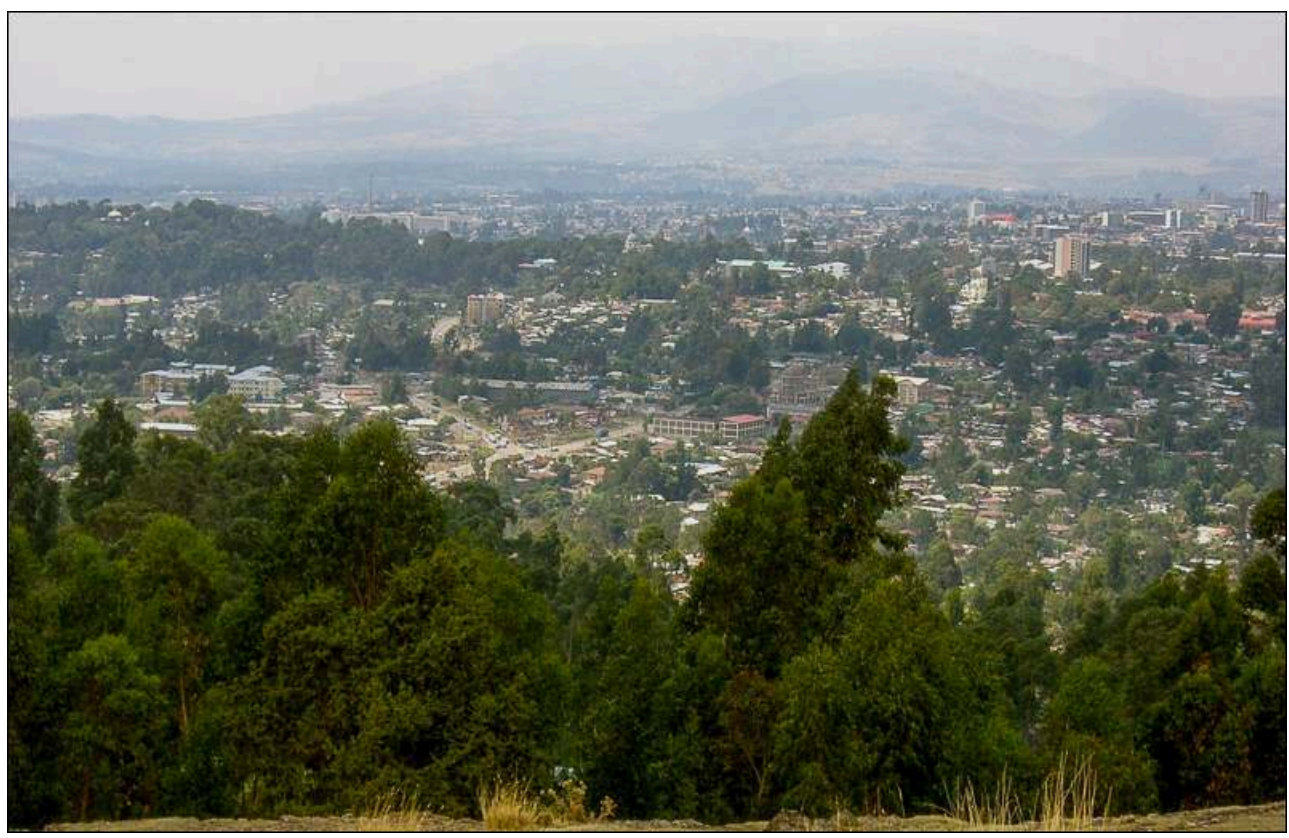

Auteur : D. Ayerbe, février 2009.

9 Au regard de la diversité des usages de cet arbre, qui recoupe à la fois des gestions gigantesques aux rendements confortables, et des appropriations plus particulières et intimes au sein des foyers privés, la qualité exotique de cette espèce semble s'estomper. Nous choisissons ici de reprendre les termes de Nancy Lee Peluso posés pour son analyse des cultures de caoutchouc en Indonésie. Elle précisait à ce sujet que les acteurs ont fait de cette culture un "produit non plus exotique mais local" (Peluso, 2012, p. 51). Si les éléments d'analyse et de contexte diffèrent, la terminologie qu'elle emploie convient à notre étude. Il semble que nous puissions arriver au même constat en ce qui concerne la place de l'eucalyptus dans la société éthiopienne.

\section{L'eucalyptus : objet de rejet}

10 Pour autant l'omniprésence de cette espèce est actuellement au cœur d'un vif débat, basé sur la volonté de décideurs urbains de la Municipalité d'enclencher une restriction des plantations d'eucalyptus. A Addis Abeba, les agents de l'Environmental Protection Authority (EPA-branche de la Municipalité), rencontrés à plusieurs occasions pour des entretiens en 2009, souhaitent une défection, du moins dans le discours, de l'eucalyptus. Les autorités reprennent avec entrain les arguments écologiques mondialement évoqués qui décrivent une espèce trop gourmande en eau et néfaste aux qualités des sols (Davidson, 1989 ; Jagger, 2000). Ces contraintes sont notamment les résultats d'une croissance rapide ( 5 ans pour atteindre une taille adulte) et du développement de rejets au niveau des souches - éléments qui ont précisément assuré le succès de la diffusion de l'espèce sur les hauts plateaux éthiopiens. Les arguments anti-eucalyptus sont basés sur des constats écologiques et portés tant par des associations ou des ONG que par les autorités publiques. Pour autant ces discours se 
posent en contradiction totale avec une réalité de terrain observée où les usages de l'eucalyptus par les habitants restent constants - même dans cette capitale qui se modernise. Ainsi, le dilemme "eucalyptus" anime de nombreuses réflexions au sein des décideurs urbains quant aux choix faits pour l'aménagement des espaces boisés de la capitale. Deux modèles de mise en valeur des sols s'affrontent: l'un s'appuie sur la nécessité de conserver des espaces productifs, quand l'autre prône la mise en place de territoires protégés et écologiquement préservés.

11 Le positionnement officiel des acteurs institutionnels, relevé lors d'entretien auprès de membres de l'EPA ou du Ministère de l'Agriculture (département Forêt) en 2009, se trouve influencé par les arguments des associations ou ONG de protection de l'environnement. Ethiopian Heritage Trust par exemple fait partie de ces associations très présentes sur le terrain, dont les actions sont médiatisées ${ }^{4}$. Cette association créée en 1992 est investie dans la conservation du patrimoine éthiopien, bâti et naturel. Elle œuvre à la réintroduction d'espèces indigènes et par la voix d'un de ses porte-paroles, et crédit scientifique - le Pr Legesse Negash ${ }^{5}$ - milite pour la suppression des plantations d'eucalyptus sur les collines de la capitale. Les arguments mobilisés sont très clairement écologiques - lutte contre l'érosion et l'assèchement des sols, même si un doux rêve de reconstituer un paysage perdu, fantasmé, n'est certainement pas à exclure... De grandes campagnes de reboisement sont régulièrement organisées, qui mobilisent membres volontaires de la société civile, hommes politiques ${ }^{6}$, et qui emploient en outre des enfants des rues pour ajouter une dimension sociale à ces programmes environnementaux. Ainsi dès l'année 1997 environ 40000 plants d'espèces indigènes ont été réintroduits dans le parc naturel $\mathrm{d}^{\prime} \mathrm{Entoto}^{7}$, territoire concédé par les autorités municipales à l'association pour la mise en place de ces projets. Depuis ces campagnes sont régulièrement organisées et surtout très médiatisées par la presse locale, même si leurs résultats concrets sur le terrain restent limités ${ }^{8}$. L'illustration 3 montre en premier plan des parcelles replantées par l'association, quand en arrièreplan les eucalyptus recouvrent toujours la majorité des collines de la capitale. 
Illustration 3 - Reboisements indigènes et plantations d'eucalyptus à Entoto

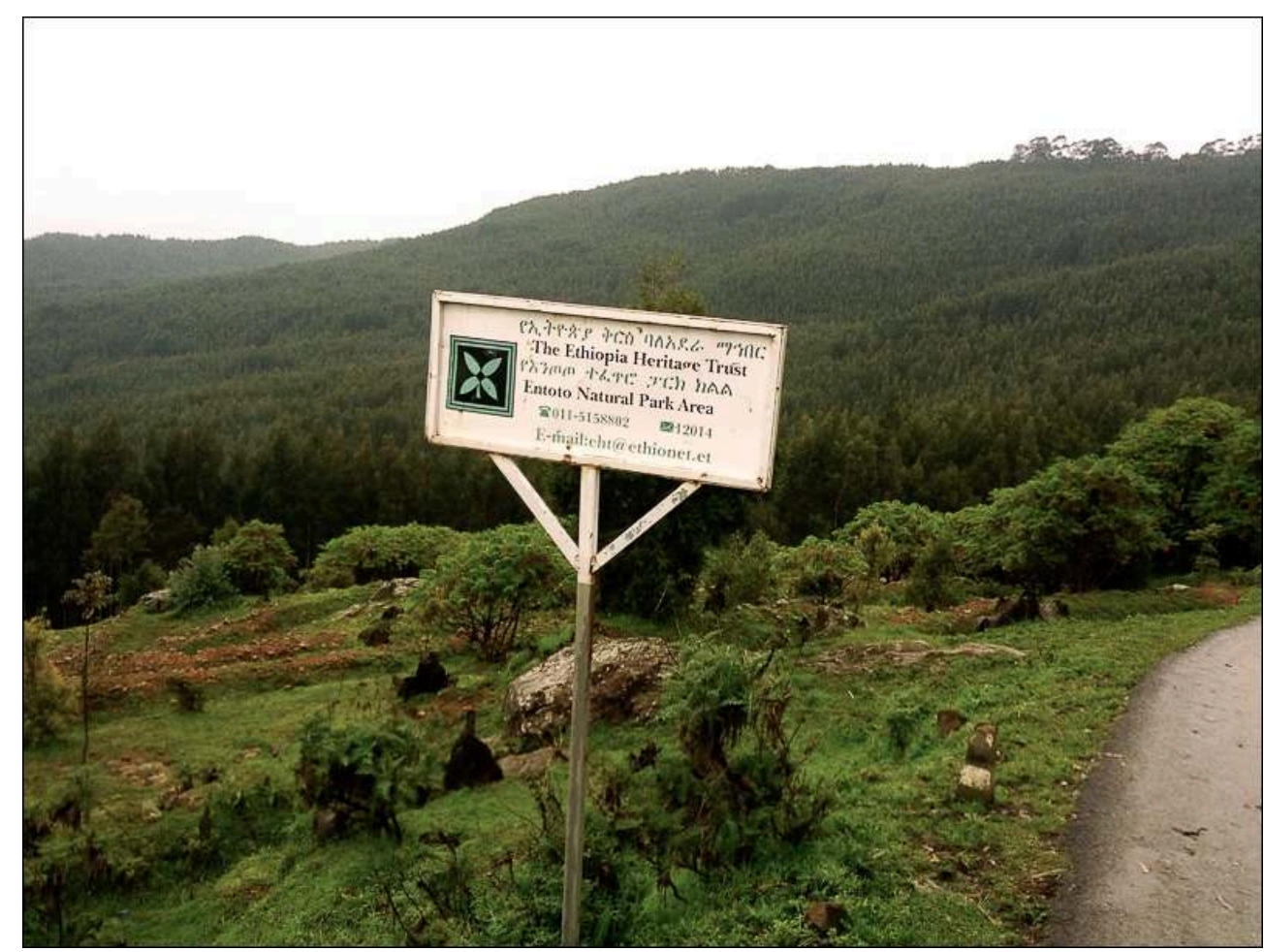

Auteur : D. Ayerbe, août 2013.

12 Ces programmes, et les discours qui les portent, expriment la volonté actuelle de réhabilitation des collines d'Addis Abeba par la suppression des plantations d'eucalyptus. Au-delà des arguments environnementaux bien compris, il semble poindre un rejet, que nous qualifierons d'identitaire, de ces plantations. En effet, dans la majorité des entretiens que nous avons eu l'occasion de mener auprès des autorités locales, comme des membres des associations de protection de l'environnement, il est stipulé que l'eucalyptus ne peut convenir aux sols éthiopiens n'étant tout simplement pas d'essence indigène. Dans ce pays très nationaliste cet argument prend sens; cet élément du paysage urbain vient à cristalliser des tensions identitaires. L'eucalyptus est et reste "l'arbre venu de la mer"9. Plus que cela, il est l'arbre de Ménélik, l'arbre qui a accompagné la conquête de l'empereur sur les territoires Oromo (Boranna), au point que ces derniers le dénomment muka qawwee, littéralement "l'arbre fusil". Selon Gamachew Magersa, professeur d'anthropologie à l'Université d'Addis Abeba, deux explications sont apportées à cette terminologie. Il peut s'agir de qualifier les effets néfastes de l'arbre sur l'environnement. Mais lui y voit surtout une façon de désigner l'arbre venu avec les fusils du conquérant, c'est-à-dire en suivant l'avancée des soldats de l'empereur Ménélik ${ }^{10}$.

13 Si cette culture exotique revêt par sa pratique une dimension locale, les symboles qu'elle véhicule dépassent largement sa dimension utilitaire, et nous renseignent sur des conflits plus larges. Le débat, basé sur des arguments écologiques, dépasse ce cadre. Et l'espèce est corrélée à un phénomène de « colonisation » (Rossi, 2001).

14 C'est pourquoi nous avons choisi de reprendre la terminologie posée par Nancy Lee Peluso, et de qualifier l'eucalyptus de " commodité problématique » aujourd'hui à Addis Abeba. Certes cet arbre est utile, et cette utilité en a fait une ressource marchande de 
poids dans l'agriculture urbaine et périurbaine (bois d'œuvre et de chauffe structurent la filière). Pour autant nous assistons en Éthiopie à la création d'un discours uniformisé de rejet de l'arbre. Porté par les décideurs urbains, il fait fi des contextes particuliers. Dans sa généralité, ce discours identifie l'espèce comme un "problème" pour l'environnement du pays.

\section{Élaboration de nouvelles modalités de régulation des plantations}

15 Ces discours de rejet de l'eucalyptus, qui aboutissent parfois à la création de territoire de préservation comme nous l'avons évoqué avec l'exemple du parc naturel d'Entoto, sont surtout un moyen de légitimer un contrôle supplémentaire pour l'accès aux espaces de production. Les plantations d'eucalyptus, pointées comme "nocives ${ }^{11}$, sont plus fortement encadrées par les autorités en charge de leur gestion, et ce aux dépens des communautés tributaires de la ressource boisée. Nous prendrons deux exemples pour illustrer ce propos et démontrer une accentuation du contrôle de l'accès à la ressource (bois d'œuvre et de chauffe) sous couvert de protection de "la nature ». Ces données sont le résultat d'enquêtes de terrain menées à Addis-Abeba entre 2008 et 2010 (et précisées en 2013). La collecte s'est faite à la fois auprès des acteurs institutionnels et des populations locales. Le croisement du discours officiel et des pratiques des habitants permettait de révéler les enjeux actuels des nouvelles modalités d'accès aux ressources. Dans les deux cas les enquêtes par entretien ont été privilégiées, beaucoup plus adaptées au contexte de l'étude et aux hypothèses posées, dans le cadre d'un travail qualitatif.

\section{Vers une gestion communautaire?}

Si les autorités se disent soucieuses des effets des plantations d'eucalyptus sur les sols, elles ne peuvent renoncer complètement à cette exploitation utile et rentable. Ainsi un mode d'organisation et de gestion communautaire de ces espaces est prôné. Cette tendance rejoint un modèle défendu par les instances internationales, qui se base sur une recherche d'intégration des populations locales afin d'assurer une gestion « durable » des ressources (Ballet, Koffi, Komena, 2009 ; Keeley, 2000).

Les plantations périurbaines sont très largement gouvernementales - soit aux mains de la Municipalité, soit aux mains du gouvernement de la région Oromo. Mais elles sont dans les faits exploitées par les populations locales qui vivent sur ces territoires. Les paysans produisent sur ces terres des perches qui serviront ensuite à la construction. Finfinnee ${ }^{12}$ Forest Development and Marketing Enterprise possède actuellement plus de 26000 ha de terres dans un rayon de $50 \mathrm{~km}$ autour de la capitale, dont une grande partie en immédiate périphérie. Sur la superficie totale plus de 17000 ha sont plantés d'eucalyptus. La lecture du plan de gestion de cette entreprise, approfondie par des entretiens auprès des membres de l'équipe administrative, nous renseigne sur les objectifs d'exploitation. L'entreprise publique ne se détache évidemment pas des contraintes de rendement; pour autant un pan plus large semble être consacré à la prise en compte des enjeux environnementaux. Les gestionnaires sont largement conscients du débat qui se noue autour de l'exploitation de l'eucalyptus et n'ont d'autres choix que de l'intégrer à leur plan d'action (puisque ces considérations sont 
admises au plus haut sommet de l'État). Ainsi, dans les dires comme dans les écrits nous retrouvons cet intérêt croissant pour une meilleure gestion des plantations d'eucalyptus, qui passe en premier lieu par un contrôle plus strict des populations locales, destructrices de la ressource ${ }^{13}$, et par extension semble-t-on comprendre, de l'environnement.

Pour enrayer ce fonctionnement néfaste, l'entreprise publique établit une série de solutions. Ces nouvelles prescriptions sont de deux modes. D'une part préserver ces territoires de populations extérieures qui viendraient collecter illégalement les ressources boisées, par un accroissement du nombre de gardes ${ }^{14}$. D'autre part assurer une gestion plus équilibrée des plantations en favorisant la gestion communautaire et en intégrant davantage les communautés locales à cet échange participatif. Les administrateurs valorisent cette gestion communautaire en présentant l'investissement escompté des populations locales dans l'exploitation forestière. Ils rejoignent surtout des prérogatives véhiculées par la plupart des ONG et associations internationales qui y voient un gage de gestion «durable » des ressources. Mais c'est aussi une façon avouée pour l'entreprise de minimiser ses frais de gestion ${ }^{15}$.

19 Même si ce type d'organisation cherche à améliorer la gestion économique et environnementale des espaces, nous ne pouvons que constater le contrôle accru du territoire qu'elle impulse. Impliquer davantage les populations locales dans la gestion peut aussi être entendu comme un moyen d'aiguiser le regard porté sur leurs pratiques. C'est en tous cas une façon de dicter un comportement jugé, pour elles, plus adéquat. D'autant que le "poids"16 du gouvernement demeure écrasant puisqu'il reste l'unique propriétaire des plantations et le seul véritablement capable d'édicter les règles d'accès aux ressources.

20 Notons enfin que ces modalités d'intégration trouvent un écho fort limité auprès des populations concernées. Les paysans interrogés, malgré toute la réserve que ce sujet pouvait imposer, ont affirmé des réticences face à ces formes de gestion participative. Une des raisons est directement liée au contexte éthiopien et aux douloureux souvenirs de la période du Derg: "The preference for individual rather than community-based user rights by farmers can partly be attributed to memories of past experience and their perceptions of land tenure, conservation and development in general» (Tarekegn, 2001, p. 63).

\section{Restriction d'accès à la ressource boisée}

21 Le contrôle des autorités s'exprime plus spécifiquement encore sur une catégorie précise - et vulnérable - d'acteurs de la filière eucalyptus. Cette filière qui réunit des acteurs divers est dominée par l'État, à la fois plus grand planteur et négociant de bois. Une série d'acteurs intermédiaires sont présents à Addis Abeba, notamment de nombreux revendeurs qui jalonnent les rues de la ville - dont les enquêtes de terrain ont montré que l'activité bénéficiait de l'essor des constructions dans la capitale. Au bas de cette pyramide, des milliers de femmes ${ }^{17}$ collectent chaque jour quelques branchages sur les plantations d'eucalyptus, et vendent leurs fagots sur les marchés de la capitale. Ce petit bois est toujours très utilisé par les additiens, notamment pour la cuisson de l'injera (galette traditionnelle). plus proches du centre-ville), mais aussi dans le parc d'Entoto. Les entretiens ${ }^{18}$ auprès 
de ces collectrices ont démontré une organisation bien rôdée. Elles sont majoritairement femmes de tisserands, venues du Sud du pays, et pratiquent cette activité de collecte dans le but d'améliorer le quotidien familial. Cela ne représente généralement pas l'essentiel des revenus du foyer mais un complément. Elles se rendent deux ou trois fois par semaine sur les plantations des hauteurs de la capitale, souvent en groupe, pour ramener quelques heures plus tard, sur leur dos, le fagot de bois qu'elles vendront aux additiens.

Leur pratique de collecte est officiellement illégale même s'il est impossible d'ignorer cette activité qui réunit chaque matin un flot continu de femmes sur les routes nord de la capitale. Leur accès aux plantations gouvernementales est contrôlé par des gardes auxquels elles doivent chaque jour payer un droit d'entrée ${ }^{19}$. Cette taxe n'a pas de valeur officielle, puisque légalement les femmes n'ont pas accès à ces espaces. Pour autant ce système est instauré et admis. Dans les faits ces collectes de bois quotidiennes sont tolérées, voire encouragées par les autorités locales. Ces dernières oscillent en effet entre deux positions à l'égard de ces femmes qui illustrent un jeu subtil dans la régulation d'accès à la ressource bois de chauffe. Officiellement le travail des "femmesfagots" est décrié par les pouvoirs publics, qui tout en dénonçant le sort misérable de ces collectrices, les accusent de détériorer les plantations par l'arrachage des branches des arbres. Plus officieusement, les administrateurs reconnaissent l'utilité de leur activité dans l'entretien des plantations. Elles ont pour mot d'ordre de ne collecter que les branchages à terre, ce qui assure un maintien des plantations étatiques.

L'accès à ces territoires exploités est conditionné par les besoins des autorités en charge de leur régulation. Un double jeu s'opère en fonction de leur positionnement. Ces contraintes d'accès variables entraînent une incertitude quotidienne pour ces femmes, et amplifient la précarité de leur situation. Cet exemple illustre le contrôle d'accès aux ressources orchestré par le gouvernement et la nuance qu'il sait apporter au regard des contraintes qui sont les siennes. Une fois encore le " problème » que constitue l'exploitation de l'eucalyptus paraît relatif, et largement lié au contexte particulier envisagé.

\section{L'appropriation d'un discours global pour légitimer une mainmise gouvernementale sur la ressource.}

\section{Les intérêts supposés des autorités dépassent le cadre environnemental}

Expliquer ces nouvelles modalités de gestion par l'objectif unique de contrôle des populations nous semblerait réducteur. Dans notre contexte d'étude l'État est très fort ; si le contrôle des populations était l'unique objectif des autorités, les modalités d'application seraient probablement plus directes. Certes ce regard posé sur les pratiques populaires sert les intérêts du gouvernement (local et à travers lui central), mais nous pensons que les explications de ces interventions et l'édification de nouvelles normes dans la pratique de l'environnement urbain sont plus subtiles et expriment des intérêts divers. Elles permettent, comme nous l'avons vu, un contrôle d'accès à la ressource. Mais au-delà de ce contrôle territorial direct, il nous semble que la fermeté des régulations souligne la volonté des pouvoirs publics de contrôler la filière eucalyptus, avec une extension allant de la ressource boisée au foncier qui la supporte. 
26 Le gouvernement est à la tête de cette filière dans les périphéries de la capitale. Il détient la très grande majorité des plantations, mais est aussi le principal consommateur de bois (notamment du bois de construction). Les autorités présentent ainsi la spécificité d'être présentes en amont et en aval de la filière bois. Elles ont donc un net intérêt à garder le contrôle de cette ressource utile et rentable. Ce contrôle leur permet en outre une subtile régulation des prix du marché. Et cela influe sur les importants volumes de bois qui arrivent dans la capitale des différentes régions éthiopiennes (notamment Guragé) ${ }^{20}$. Car hors d'Addis Abeba, le commerce du bois échappe davantage aux autorités publiques.

Les perspectives de développement et de rentabilité projetée sur l'eucalyptus ne sont certainement pas étrangères à ce contrôle accru. En effet la transformation de la matière première, en pâte à papier, est absente du développement actuel de la filière mais est évoquée comme un axe de prospection possible ${ }^{21}$. Même si ces visées restent pour l'heure chimériques, elles traduisent la rentabilité réelle et supposée de la ressource. Il nous semble, au regard des recherches de terrain, que cet aspect constitue un des facteurs explicatifs de l'encadrement de la ressource auquel nous assistons.

Mais surtout les pouvoirs publics cherchent à conserver le contrôle de cette ressource utile au développement urbain. Si l'eucalyptus a accompagné le développement d'Addis Abeba et a soutenu la croissance urbaine en fournissant aux populations le bois nécessaire à leur implantation, il reste aujourd'hui encore un élément important de la construction urbaine (Gascon, 2009). Et ce pour deux raisons, d'une part par les recettes budgétaires que son commerce fournit à la Municipalité, et d'autre part comme matériau de construction utile au développement urbain. L'édification de maisons en bois est maintenant interdite, mais les échafaudages sont eux presque toujours montés en bois d'eucalyptus. Au rythme effréné de la construction urbaine dans la capitale, cet usage du bois n'est pas négligeable. Et l'arbre, de façon détourné, reste un élément de l'essor urbain.

29 Ensuite, le contrôle des espaces boisées périphériques peut se comprendre comme le verrouillage d'une potentielle réserve foncière pour les autorités municipales. La croissance urbaine et l'avancée du bâti exercent une pression sur les terres en immédiate périphérie. Dans ce contexte, il peut être judicieux pour le gouvernement de conserver des réserves foncières, aujourd'hui plantées d'arbre mais potentiellement constructibles. Pour l'instant l'avancée du bâti, très remarquable à Addis Abeba depuis une dizaine d'années, concerne assez peu les périphéries nord de la capitale, marquées par un relief notable ${ }^{22}$. Pour autant, un léger recul des plantations est enclenché sur les collines de Yeka, qui deviennent un lieu de résidence d'une classe aisée de population, où percent quelques villas modernes.

30 Enfin, cette régulation des espaces plantés, influencée par des discours de préservation de l'environnement, atteste d'une force de commandement malgré tout centralisée, qui édicte des préceptes globalisés aux différents échelons de cet État fédéral. Car à travers ces exemples à échelle locale, émergent les orientations générales des autorités en matière de pratiques environnementales. Les régulations dictées au plus haut sommet de l'État, sont diffusées sur le terrain à échelle fine, par un système d'encadrement coordonné. Les politiques municipales en matière d'environnement expriment les décisions prises au niveau national. 


\section{Les pratiques de l'environnement au service de l'image de la capitale}

Cette rhétorique du rejet de l'eucalyptus chez les acteurs locaux traduit l'acceptation d'un discours global, empreint d'un souci de préservation de l'environnement, à même de dicter de nouvelles pratiques.

L'acceptation de ce discours nous semble avoir deux piliers. D'une part la surreprésentation d'ONG et d'associations de protection de l'environnement dans la capitale éthiopienne, qui joue un rôle dans la diffusion de ces préceptes. Beaucoup de ces structures sont des organisations internationales (nord-américaines, allemandes, hollandaises etc. - nous avons eu l'occasion d'échanger avec diverses d'entre elles). Certaines de ces structures sont de renommée internationale (comme SOS Sahel), d'autres sont plus confidentielles. Mais leur enracinement est solide. Cette forte présence est le résultat des stéréotypes "misérabilistes" associés à l'Éthiopie suite aux grandes famines des années 1980 et qui ont attiré la venue de nombreuses ONG. Mais le foisonnement de ces activités est aussi le fait d'une communauté internationale importante. Addis Abeba est le siège de l'Union Africaine. A ce titre elle abrite de nombreuses ambassades et diverses délégations étrangères y séjournent. Les Nations Unies y sont également implantées. Cette internationalisation des acteurs joue dans le développement d'un argumentaire global sur les questions de gestion de l'environnement. Les travaux des structures non institutionnelles forgent alors la diffusion de ce discours global à l'échelle du pays.

En outre l'État, par l'intermédiaire de la Municipalité, cherche à développer un discours consensuel, qui lui apporte une reconnaissance et une assise internationales. La modernisation $\mathrm{du}$ paysage urbain qui est enclenchée s'opère aussi par une revalorisation des espaces verts, et des espaces boisés. Les autorités encouragent une réhabilitation qui puisse œuvrer pour l'amélioration de l'image de la ville. À travers sa dimension diplomatique internationale, les décideurs urbains rêvent de positionner la ville dans le rang tant convoité des métropoles mondiales. Les prises en compte croissantes de l'environnement urbain s'intègrent à ce mouvement. L'étude du Master Plan et les discussions avec les acteurs officiels nous montrent que les espaces boisés sont inclus dans cette dynamique. Les pratiques qui y contribuent sont contrôlées dans l'espoir de faire correspondre ces touches de vert aux exigences d'une grande capitale qui doit se positionner en ville «durable» si elle veut compter sur la scène internationale. Les projections envisagent d'accroître encore les espaces de forêt. Le dernier Master Plan (toujours valable) prévoit de consacrer $12500 \mathrm{~h}$ du territoire à la forêt (Haile, 2004; Horst, 2006). Mais les décideurs urbains hésitent encore sur la fonctionnalité à attribuer à ces espaces forestiers, partagés entre volonté de développer des espaces récréatifs, et la nécessité de conserver des espaces productifs. Prérogatives globales et enjeux locaux se rencontrent sur ces territoires.

\section{Conclusion}

34 Le contrôle des plantations d'eucalyptus à Addis Abeba n'est pas un fait nouveau puisque ces espaces sont depuis des dizaines d'années aux mains des autorités. La spécificité actuelle réside dans une volonté de régulation d'accès à la ressource boisée fondée sur une série d'arguments écologiques. 
souci de préservation de l'environnement, les pouvoirs locaux produisent de nouvelles modalités de contrôle des espaces plantés. Il s'agit d'évincer des pratiques populaires néfastes au profit d'une gestion plus appropriée et davantage respectueuse de l'environnement, car mieux encadrée par les autorités. Fondée sur un argumentaire global, cette dynamique nous semble aussi traduire les intérêts des pouvoirs publics à une échelle plus fine. En œuvrant pour une préservation écologique des territoires, les autorités assoient aussi leur domination sur ces espaces et s'assurent un contrôle d'accès et d'utilisation de la ressource, doublé de la réserve foncière que constituent ces plantations. La mainmise sur la filière bois constitue un élément non négligeable du développement urbain de la capitale. En effet l'eucalyptus demeure un élément de soutien de l'essor urbain actuel. Il représente toujours un matériau de construction au service de la capitale qui se densifie. Mais cette exploitation fournit surtout une culture de rente pour le gouvernement.

L'actuelle gestion des plantations d'eucalyptus à Addis Abeba repose sur un équilibre subtil. Les autorités s'approprient et énoncent des préceptes environnementaux globaux, qui servent des intérêts écologiques locaux, et permettent surtout un contrôle plus strict de la ressource.

\section{BIBLIOGRAPHY}

Amare G., 2003. Eucalyptus Farming in Ethiopia: the Case for Eucalyptus Woodlots in the Amhara region. In Proceedings of the conference on natural resources degradation and environmental concerns in the Amhara National Regional State, Ethiopia: Impact on food security, Bahir Dar, Ethiopia.

Ballet J., Koffi K., Komena K., 2009. La soutenabilité des ressources forestières en Afrique subsaharienne francophone : quels enjeux pour la gestion participative? Mondes en Développement, $\mathrm{n}^{\circ} 148$, p. 31-46.

Belachew Y., 2010, Urban Land Lease Policy of Ethiopia. Case study on Addis Ababa and Lease towns of the Amhara National Regional state. FIG Congress 2010. Facing the Challenges - Building the Capacity. Sydney, Australia, 11-16 April 2010.

Berlan E., 1963. Addis-Abeba la plus haute ville d'Afrique. Étude géographique, Grenoble, Allier.

Carruthers J., Robin L., Hattingh J. P., Kull C. A., Rangan H., van Wilgen B. W., 2011. A native at home and abroad: the history, politics, ethics and aesthetics of Acacia. Diversity and Distributions, 17 (5), p. 810-821.

City Development Plan 2001 - 2010, Executive summary. Addis Ababa in action: Progress Through Partnership, Addis Ababa.

Couret D. et al., 2011. L'introuvable ville durable. In N. Mathieu et Y. Guermond, La ville durable, du politique au scientifique, Editions Quæ «Indisciplines », p. 47-56.

Davidson J., 1989. The Eucalypt Dilemma, Arguments for and against Eucalypt Planting in Ethiopia. Addis Ababa, Forestry Research Center, Seminar notes series $n^{\circ} 1$.

EchoGéo, 29 | 2014 
Fekerte H., 1991. Women Fuelwood Carriers in Addis Ababa and the Peri-Urban Forest. Geneva, International Labour Office, Report to the Government of Norway, the International Development Research Centre and the National Urban Institute.

Gallais J., 1989. Une géographie politique de l'Éthiopie. Le poids de l'État. Paris, Economica, Liberté sans frontières.

Gascon A., 1999. Addis Abäba, la plus haute capitale d'Afrique. In A.M Frérot (dir.), Les Grandes villes d'Afrique, Paris, Ellipses.

Gascon A., 2009. Les Éthiopiens, des hommes qui plantent des arbres . In B. Hirsch, B. Roussel, (dir.), Le Rift est-africain. Une singularité plurielle, IRD Éditions, Marseille, p. 205-211.

Gascon A., 2008. Shining Ethiopia : l'Éthiopie post-communiste du nouveau millénaire. Autrepart, $\mathrm{n}^{\circ} 48$, p. $141-152$.

Gautier D., Benjaminsen T., 2012. Environnement, discours et pouvoir. L'approche Political ecology. Versailles, Editions Quae.

Government of Ethiopia, Natural Resources Conservation and Development Main Department, 1987. Addis - Bah forestry development project. Socio - Economic study. Final Report, Vol. 3: Addis Ababa : new plantation area, ULG Consultants limited.

Haile Sellassie Sebhatu, 2004. Urban forestry: its challenges and development strategies - The case of Addis Ababa. In Proceedings of the Public Meetings on Integrated Forest Policy Development in Ethiopia, Forum For Environment, Addis Ababa.

Hoben A., 1995. Paradigms and politics: The cultural construction of environmental policy in Ethiopia. World Development, 23(6), p. 1007-1021.

Horst A., 2006. Rehabilitation of urban forests in Addis Ababa. Journal of the Drylands, 1(2), p. 108117.

Jagger P., Pender J., 2000. The role of trees for sustainable management of less-favored lands: the case of eucalyptus in Ethiopia. Environment and Production Technology Division, Discussion Paper $n^{\circ} 65$, International Food Policy Research Institute, Washington, D.C.

Keeley J., Scoones I., 2000, Knowledge, power and politics: the environmental policy-making process in Ethiopia. The Journal of Modern African Studies, 38, p. 89-120.

Metzger P., 1994. Contribution à une problématique de l'environnement urbain. Cahiers des Sciences Humaines, 30 (4), Orstom, p. 595-679.

Metzger P., Couret D., 2002. La ville durable côté Sud : entre utopies et pratiques. In J.Y. Martin (dir.), Développement durable. Doctrines, pratiques, évolutions, IRD Éditions, Objectifs Suds, p. 161-181.

Metzger P., Couret D., 2010. Vulnérabilité et pauvreté en milieu urbain : réflexions partir des villes du Sud. In O. Coutard , J.-P. Lévy (dir.), Écologies urbaines, Paris, Economica-Anthropos.

Mugélé R., 2013. Enjeux et conséquences de la réglementation sur le bois-énergie au Tchad. EchoGéo [En ligne], 26 | 2013, mis en ligne le 19 décembre 2013. URL : http://echogeo.revues.org/ 13620 ; DOI : 10.4000/echogeo.13620

Oromia Regional National Government, Finfinnee Forest Development and Marketing Enterprise, 2005. Forest Resources Development and Utilization Management Plan, for the Period of July 2005 - June 2011, Addis Ababa, Finfinnee. 
Peluso N., 2012. Situer les political ecologies : l'exemple du caoutchouc. In Gautier D., Benjaminsen T., Environnement, discours et pouvoir. L'approche Political ecology, Versailles, Editions Quae, p. 37-63.

Rossi G., 2001. L'Ingérence écologique - Environnement et développement rural du Nord au Sud. CNRS Editions, coll. Espaces et milieux.

Tarekegn Y., 2001. Enclosing or 'Individualising' the 'Commons'? The Implementation of two User-rights Approaches to 'Communal' Areas Management in Post-Derg Northern Ethiopia. In A. Pankhurst (dir), Natural Resource Management in Ethiopia, Proceedings of the Workshop organised by Forum for Social Studies in collaboration with the University of Sussex, Addis Ababa, 9 February 2001, Forum for Social Studies, Addis Ababa, p. 51-84.

\section{NOTES}

1. La Municipalité s'inscrit par exemple dans les prérogatives fixées par les objectifs du Millenium des Nations Unies et par l'Agenda 21.

2. Peluso, p. 45.

3. La sélection des sites de programmes de reboisement est détaillée dans Addis - Bah forestry development project. Socio - Economic study (1987).

4. Notamment par la présence de soutien de renoms, comme une des petites filles d'Hailé Sélassié, très impliquée dans cette association et qui la représente lors d'évènements à visée internationale.

5. Professeur de biologie à l'Université d'Addis Abeba. Entretien en février 2008.

6. Informations recueillies lors de deux entretiens avec des membres du personnel administratif d'EHT - 2009.

7. Situé sur l'illustration 1.

8. Le parc d'Entoto recouvre un peu plus de 1000 ha, mais une infime partie est replantée en espèces indigènes (les estimations sont de l'ordre de $10 \%$ ).

9. Bar Zaf en amharique.

10. Propos recueillis lors d'entretiens en 2009.

11. Selon, par exemple, un manager du parc d'Entoto - Entretien à Addis Abeba en octobre 2009.

12. Nom Oromo pour Addis Abeba.

13. "The local communities have been seen as destroyers of the forest" in Finfinnee Management Plan, p. 54.

14. "The Enterprise has been forced to employ 600 forest guards to protect from illegal cutters", ibid.

15. Finfinnee Management Plan, p. 55.

16. En référence au titre de l'ouvrage de Jean Gallais : Une géographie politique de l'Éthiopie. Le poids de l'État.

17. L'association Women fuelwood carriers donne pour estimation le nombre de 10000 femmes à Addis Abeba.

18. Environ 30 femmes ont été interrogées lors d'entretiens allant de $15 \mathrm{~min}$ à $40 \mathrm{~min}$ selon les disponibilités des enquêtées.

19. Un ou deux birrs (monnaie locale) quand le fagot est revendu autour de douze birrs.

20. Réalisation d'enquêtes par observation aux 5 postes de douanes d'Addis Abeba, qui ont permis d'identifier les principaux flux de bois vers la capitale.

21. Notamment par des entreprises implantées à Addis Abeba, et qui aujourd'hui utilisent l'eucalyptus pour la fabrication de meubles en aggloméré.

22. Environ $500 \mathrm{~m}$ de dénivelé. 


\section{AUTHOR}

\section{DELPHINE AYERBE}

Delphine Ayerbe, delphineayerbe@hotmail.com, est doctorante en géographie, membre de l'UMR Prodig, Université Paris 1. 